\title{
Unraveling the multistimuli responses of a complex dynamic system of pseudopeptidic macrocycles
}

\author{
Angel M. Valdivielso, ${ }^{[a]}$ Francesc Puig-Castellví, ${ }^{[b]}$ Joan Atcher, ${ }^{[a]}$ Jordi Solà, ${ }^{[a]}$ Romà Tauler ${ }^{[b]}$ and \\ Ignacio Alfonso*,[a]
}

Dedicated to Prof. Vicente Gotor on the occasion of his $70^{\text {th }}$ birthday

\begin{abstract}
Dynamic combinatorial libraries (DCLs) are excellent benchmark models to study the stimuli-responsiveness of chemical networks. However, increasingly complex systems are difficult to analyze with simple data analysis methods, because many variables and connections must be considered for their full understanding. Here we propose the use of multivariate data analysis methods to bisect the evolution of a complex synthetic dynamic library of pseudopeptidic macrocycles, containing side chains with charges of different sign. Several stimuli (ionic strength, $\mathrm{pH}$ and the presence of a biogenic polyamine) were applied to the same dynamic chemical mixture, and the adaptation of the whole system was characterized by HPLC and analyzed with Principal Component Analysis (PCA) and Multivariate Curve Resolution-Alternating Least Squares (MCRALS) methods. Both multivariate data analysis chemometric approaches are an excellent combination to extract both qualitative and semi-quantitative information about the adaptive process of the library upon the action of each stimulus. The resolution of the system with these chemometric tools proved to be especially useful when two inter-connected stimuli were combined in the same dynamic system. Our results demonstrate the utility of these two approaches for the analysis of complex dynamic chemical systems and open the way toward the application of these powerful tools in the emergent field of Systems Chemistry.
\end{abstract}

\section{Introduction}

Complexity is a fascinating concept that has always attracted the scientific community, with important implications in many different areas of knowledge. ${ }^{[1]}$ For instance, it is pivotal for the understanding of interconnected networks like the cell metabolism, ${ }^{[2]}$ the economic markets, ${ }^{[3]}$ the biological ecosystems ${ }^{[4]}$ or the social networks. ${ }^{[5]}$ However, the historical reductionism of chemists has delayed the advances in the research on complexity from the chemical point of view. ${ }^{[16,6]}$ Only after the recent development of potent and sensitive analytical techniques for treating complex mixtures, in

[a] Dr. A. M. Valdivielso, Dr. J. Atcher, Dr. J. Solà, Dr. I. Alfonso Department of Biological Chemistry and Molecular Modeling IQAC-CSIC

Jordi Girona 18-26, E-08034 Barcelona, Spain.

E-mail: ignacio.alfonso@iqac.csic.es

[b] Mr. F. Puig-Castellví, Prof. Dr. R. Tauler

Department of Environmental Chemistry

IDAEA-CSIC

Jordi Girona 18-26, E-08034 Barcelona, Spain.

Supporting information for this article is given via a link at the end of the document.((Please delete this text if not appropriate)) combination with powerful computational tools, the chemists have started to investigate complex chemical networks. ${ }^{[7]}$ This has crystallized in a new area of research named Systems Chemistry. ${ }^{[8]}$ Remarkably, the understanding of the evolutionary patterns in these chemical systems upon the action of meaningful stimuli is fundamental in prebiotic chemistry and for the understanding of the origin of life. ${ }^{[9]}$ Moreover, the complexity itself is interesting, since the network of interconnected species can show abilities that are different from the ones exerted by their components (the so-called emerging properties). ${ }^{[7,10]}$

Among the different chemical systems described to date, dynamic combinatorial libraries represent an appealing network for several reasons. ${ }^{[11]}$ The first one is that they operate under equilibrium, which allows extracting thermodynamic properties (relative stabilities) of the different species from the library behavior. ${ }^{[12]}$ On the other hand, since the members of the library are connected through reversible reactions, the mixture itself is adaptive as a whole, exerting error correction capabilities. ${ }^{[13]}$ These features have led to the use of dynamic combinatorial libraries (DCLs) for the discovery of new molecules with unexpected structures, ${ }^{[14]}$ efficient receptors ${ }^{[11 a,}$ 15] and catalysts, ${ }^{[16]}$ new materials ${ }^{[17]}$ or even ligands for biomolecules as potential drugs. $\left.{ }^{[10 a}, 18\right]$ Therefore, DCLs have found interesting applications in different fields of chemical research. On the other hand, these mixtures can provide information about molecular properties within a chemical network, with important conceptual consequences. In this regard, the study of bio-inspired dynamic libraries is especially attractive because it allows proposing parallelisms with clearly more complex biological networks where relationships are more difficult to establish than in a closed and synthetic system. ${ }^{[1 \mathrm{~b}, 6,10 \mathrm{c}, 19]}$

Within our research interest in constitutional dynamic chemistry, ${ }^{[19 a}$, 20] we have recently described a DCL of macrocyclic pseudopeptides able to respond to a meaningful biological stimulus: the increase of the salt concentration. ${ }^{[21]}$ During that study and for the suitable description of the system as a whole, we carried out the corresponding dynamic deconvolution ${ }^{[22]}$ up to the minimal sub-libraries able to adapt to the same trigger. This top-down approach permits a full thermodynamic description of the complex system and even the design of ad hoc libraries with tailored relationships between the components (cooperative or competitive). However, this approach is not practical for applying different stimuli over the same library, since the experimental dynamic deconvolution must be done for each case. Accordingly, here we propose a different approximation to the problem.

In this work, the adaptive behavior of the DCL has been studied using statistical and multivariate data analysis chemometric methods. ${ }^{[23]}$ These chemometric methods have been previously used to analyze DCLs for pattern-based 
sensing of different analytes, mainly by the groups of Anslyn, ${ }^{[24]}$ Severin ${ }^{[25]}$ and Matile, ${ }^{[26]}$ among others. ${ }^{[27]}$ Our approach here is conceptually different, since we have used the chemometric tools to understand the responses of complex DCLs to several triggers. To achieve that, three different stimuli were applied. The first one is the ionic strength, which had been thermodynamically characterized by us for the same library. ${ }^{[21]}$ This allows comparing the conclusions extracted from the two alternative approaches. Secondly, the combination of two interdependent stimuli (i.e. $\mathrm{pH}$ and ionic strength) was applied as a benchmark example of multistimuli-responsive dynamic complex system. Finally, an organic polyelectrolyte with biological relevance, the spermine alkaloid, was used as a template. Preliminary work suggested that this polycationic molecule interacts with some of the virtual members of the DCL, ${ }^{[28]}$ and this must be reflected in the library properties. Besides, the polycationic substrate concomitantly increases the ionic strength of the medium and we propose a chemometric approach to bisect the two otherwise inseparable stimuli (binding and ionic strength increase).

\section{Results and Discussion}

\section{Design and generation of the complex dynamic chemical system}

For the study of the effect of different stimuli, we used a previously described dynamic mixture of pseudopeptidic macrocycles (Scheme 1). ${ }^{[1]}$ This was generated by the combination of six pseudopeptidic building blocks (BBs) bearing two thiols for the formation of the corresponding disulfides, which represent a well-known dynamic covalent bond in aqueous media. ${ }^{[29]}$ The dynamic mixture was mainly composed by 21 macrocyclic dimers and the minor presence of some trimers (as confirmed by ESI-MS). For simplicity, we will focus our discussion on the main dimeric members of the library. The adaptive properties of the system sourced from the peptide-like information contained in the two amino acids present in each BB To get responsiveness to ionic species (medium or substrates) and to the $\mathrm{pH}$ value, we used BBs with carboxylic acids (from Asp (1a) and Glu (1b)) or amine groups (from Orn (1e) and Lys (1f)), as well as innocent polar side chains (Ser (1c) and Asn (1d)). Thus, different protonation degrees of some of the side chains would implement different electrostatic charges in the library members. As in our previous work, we have classified the members of the library (macrocyclic dimers) into six different families attending to their charge at neutral $\mathrm{pH}$ (Scheme 1). Thus, we distinguished the tetra-charged dimers containing four anionic carboxylates $\left(F_{-,-)}\right.$or four cationic ammonium groups $\left(F_{+,+}\right)$, the neutral species formed by either neutral BBs $\left(F_{0,0}\right)$ or a combination of positive and negative ones $\left(F_{+,-}\right)$and those combining a dicharged $B B$ with a neutral one $\left(F_{+, 0}\right.$ and $\left.F_{0,-}\right)$. Besides, these families were readily separated during the chromatographic analysis by HPLC (see Figure 1). Thus, this chemical system contains species that are sensitive to changes in the $\mathrm{pH}$ or to the presence of charged molecules or ions. The corresponding sensitivity must be expressed in the library composition and, therefore, we aimed to monitor its changes upon applying different stimuli.

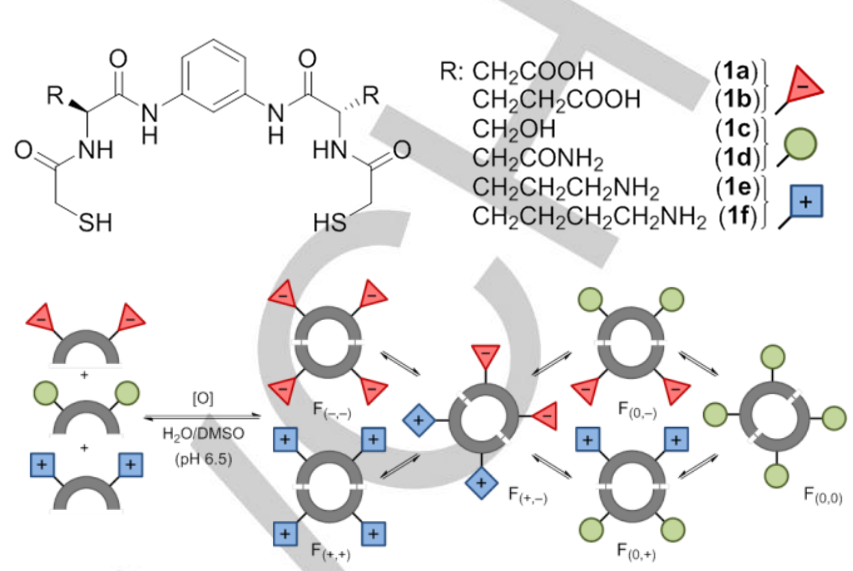

Scheme 1. Chemical structures of the corresponding BBs (1a-f) and schematic representation of the dynamic mixture generated by the disulfide formation in solution, rendering the corresponding six different families classified by their charge at neutral $\mathrm{pH}$.

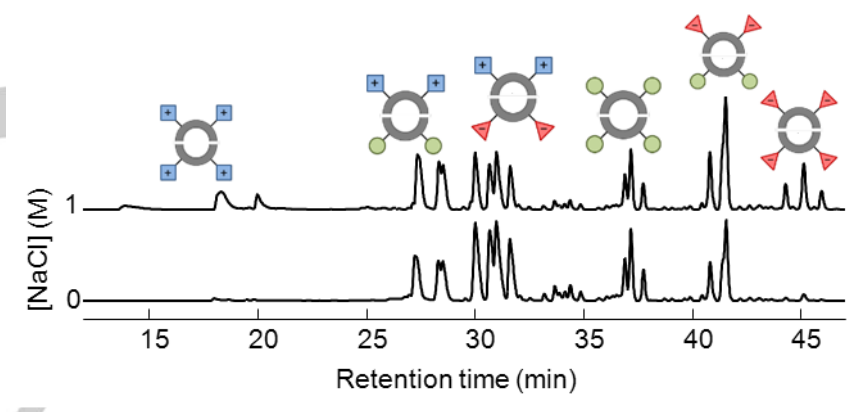

Figure 1. Effect of the salt content on the pseudopeptidic macrocycles mixture of the DCL. HPLC traces of the DCL of pseudopeptidic macrocycles at $0 \mathrm{M}$ $\mathrm{NaCl}$ (lower trace) and at $1 \mathrm{M} \mathrm{NaCl}$ (upper trace).

\section{Chemometric analysis of the DCLs}

The application of several stimuli to a complex system requires a good analytical tool to rapidly and efficiently determine their effect. This is not an easy task when multi-stimuli complex dynamic networks are studied. Here we have applied chemometric approaches to the problem, namely Principal Component Analysis (PCA $)^{[30]}$ and Multivariate Curve Resolution-Alternating Least Squares (MCR-ALS) ${ }^{[31]}$. The PCA analysis has served to perform a preliminary estimation of the most important effectors that explain the variance of the library adaptation to the stimuli by locating the different samples in a orthogonal vector subspace defined by the principal components (see experimental section for mathematical details). Thus, the scores plot would give information about the location of the samples in this vector subspace, while the representation of the loadings in this vector subspace will indicate the relative influence of the stimulus of each member of the DCL in the corresponding principal components. PCA analysis was carried 
out using the peak areas regarding each one of the six macrocycle families. In addition, data were auto-scaled prior PCA analysis.

On the other hand, although PCA provides a meaningful interpretation of the adaptive behavior of the DCL, it provides a minor semi-quantitative abstract picture. This can be improved using the MCR-ALS method, which describes the original dataset by means of a set of components via maximizing the total explained variance using more natural constraints than in PCA, like non-negativity instead of orthogonality constraints. Accordingly, the MCR-ALS method produces more feasible (from a physicochemical point of view) and interpretable solutions. In our specific case, the components resolved by MCR will represent the different states of the library associated to the absence or the presence of the stimuli. Thus, each state is defining the singular library composition that reflects a particular responsiveness of the species to the effector. Besides, the contribution of each state in every sample is also resolved in the scores obtained by MCR-ALS, which illustrates the semiquantitative evolution of the system through the action of the stimulus. For the MCR-ALS analysis, we used the raw HPLC traces (after simple manipulation, see experimental section) that allow chromatographic resolution of the species within each state of the DCLs. The transition between states of the library can be easily determined by the difference between the respective loadings obtained by MCR-ALS.

\section{lonic strength as an environmental stimulus}

The nature of the side chains of the pseudopeptidic macrocycles of the DCL makes the dynamic system prone to adapt to the presence of salt, by electrostatic reasons. This has important biological implications in terms of evolutionary patterns and it has been previously discussed within the field of dynamic constitutional chemistry. ${ }^{[19 a]}$ To study the effect of the salt content in our library, we prepared mixtures of the BBs $(0.5 \mathrm{mM}$ each) in $40 \mathrm{mM}$ aqueous bis(2-hydroxyethyl)aminotris(hydroxymethyl)methane (bis-Tris buffer, $\mathrm{pH}$ 6.5) with $25 \%(\mathrm{v} / \mathrm{v}) \mathrm{DMSO}^{\left[{ }^{[32]}\right.}$ The disulfide formation and exchange was performed in different media containing $0,0.5,1.0$, and $2.0 \mathrm{M}$ $\mathrm{NaCl}$, respectively. Two independent batches of reactions were prepared (labelled as $a-b$ ) to test the ability of the method in checking the reproducibility of the process. A total of 8 samples (4 concentrations, 2 batches) were measured by HPLC (see methods). The resulting DCL data set containing the 8 corresponding chromatograms will be referred hereafter as the $\mathrm{HPLC}_{\mathrm{NaCl}}$ data set. Figure 1 shows the HPLC traces of an equilibrated library in the absence and in the presence of $\mathrm{NaCl}$ (1 $\mathrm{M}$ in the figure). The simple visual inspection of the changes observed in the chromatograms showed a big impact of the salt on the library composition. As already mentioned, we have previously observed this effect and we were able to analyze the different equilibria implicated in the process by dynamic deconvolution. ${ }^{[21]}$ Here we aimed to use chemometric tools to extract information of the process, without the need of running additional reactions with simplified sub-libraries.

PCA results: As shown in the corresponding PCA scores and loadings plots ( $P C A$, Figure $2 \mathrm{~A}$ and $2 \mathrm{~B}$, respectively), the first two principal components (PCs) already captured the $97.69 \%$ of the explained variance. This revealed that the salt-dependent adaptive process of this apparently rather complex dynamic library can be explained by two components (patterns) describing the major changes produced by ionic strength. The scores on the first principal component ( $\mathrm{PC} 1$, already explains the $83.66 \%$ of the data variance) mostly show changes produced by increasing the salt concentration (Figure 2A). In this $\mathrm{PC} 1$, scores of samples without $\mathrm{NaCl}$ have negative values (left), whereas an increase of $\mathrm{NaCl}$ concentration produces the scores of the samples progress to positive values (right). Also in Figure $2 \mathrm{~A}$, scores distribution of samples on the second component (PC2, 14.03\% of the explained variance) could be attributed to sample batch differences unrelated to the salt effect (see below). In other words, with PCA, it is possible to separate the variances linked to the salt concentration effect (in PC1) from the variance related to the batch effect (PC2).
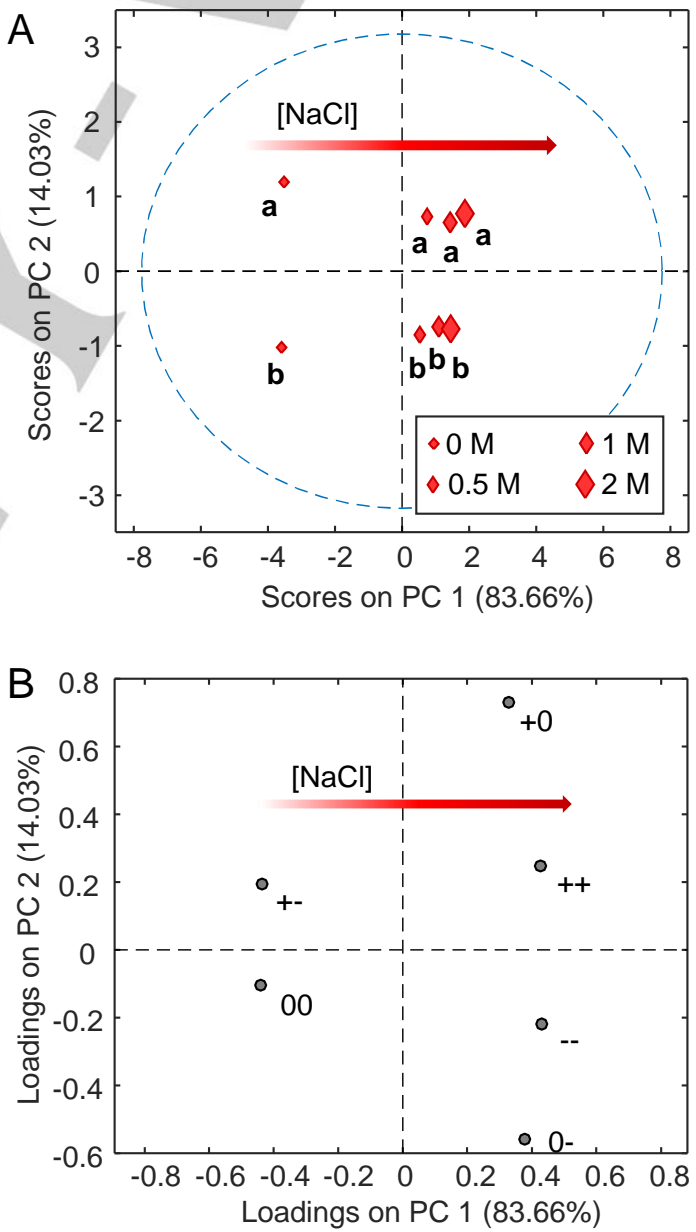

Figure 2. (A) Plot of the sample scores projected on the two first components obtained by PCA analysis of HPLC peak areas of the six macrocycle families in DCLs at different salt contents $\left(\mathrm{HPLC}_{\mathrm{NaCl}}\right.$ data set). (B) Plot of the variable loadings projected on the two first components obtained by PCA analysis of the $\mathrm{HPLC}_{\mathrm{NaCl}}$ data set. 
Regarding the variations of the loadings in the plot of Figure 2B, the increase of salt concentration favors the formation of dimers containing one neutral and one di-charged species $\left(F_{+, 0}\right.$ and $\left.F_{0,-}\right)$ and, specially, the corresponding tetra-charged macrocycles $\left(F_{+,+}\right.$and $\left.F_{-,-}\right)$, whereas neutral dimers $\left(F_{0,0}\right.$ and $\left.F_{+,-}\right)$are disfavored. This behavior can be interpreted from the loadings distribution in the plot of Figure $2 \mathrm{~B}$, since neutral dimers are represented by negative PC1 loadings, whereas the other four dimer families are linked to positive PC1 loadings. Since tetracharged dimers are linked to higher loadings than di-charged dimers, they are preferentially amplified by the salt.

Interestingly, by studying more in detail the loadings of the macrocycle family in Figure $2 \mathrm{~B}$, we observed that $\mathrm{PC} 1$ loadings for each of the pairs $F_{+,+}$and $F_{-,-}$(tetra-charged), $F_{+, 0}$ and $F_{0,-}$ (dicharged), and $F_{+,-}$and $F_{0,0}$ coincided, indicating that the effect of salt triggers the same adaptive response regardless of the type of the charge (positive or negative). We must keep in mind that this statement refers to auto-scaled values, and not to concentrations or fold-changes. In auto-scaling, the measured areas are corrected by the mean concentration of each dimer family and divided by their standard deviation. Thus, when autoscaling, dimer families with different relative abundances can present the same adaptive response. This is the case for the neutral species $\left(F_{+,-}\right.$and $\left.F_{0,0}\right)$, although they are not equally favored in terms of entropy and electrostatic interactions $\left(F_{+,-}\right.$is found in the range of $20-40 \%$, whereas $F_{0,0}$ is found in the range of $2-3 \%$ ).

In addition, since positively charged $\mathrm{F}_{+,+}$and $\mathrm{F}_{+, 0}$ are located in the positive side of PC2 loadings and negatively charged $F_{,}$, and $F_{0,-}$ are located in the negative side of PC2 loadings (Figure 2B), it implies that samples prepared during the first batch (subset a), associated to positive PC2 scores (Figure 2A), contained slightly higher amounts of positive BBs (1e and 1f) than the other batch (subset $b$ ). An experimental explanation for these differences relies on the fact that $\mathbf{1 e}$ and $\mathbf{1 f}$ were isolated as highly hygroscopic TFA salts leading to some inaccuracy in weighting these BBs for different batches of reactions. These results were deduced from the only application of PCA. In contrast they were not so obvious from the direct analysis of the raw dataset. In fact, in the raw data, differences between batches were $\sim 1.5 \%$, which could have been overlooked.

In order to distinguish whether the dynamics related to salt effect observed in PC1 are a consequence of the specific ion composition or just due to the ionic strength, we prepared an additional set of libraries maintaining the ionic strength (equivalent to $1 \mathrm{M} \mathrm{NaCl}$ ) but varying the salt composition. The different salts consisted of both monovalent and divalent ions ( $\mathrm{NaCl}, \mathrm{MgCl}_{2}, \mathrm{MgSO}_{4}$ and $\mathrm{Na}_{2} \mathrm{SO}_{4}$ ). To increase the sample pool, salts were combined in different proportions (see Supplementary Table S1), rendering the same ionic strength as $1 \mathrm{M} \mathrm{NaCl}$. As shown in the PCA plot in Figure S1, no sample differences related to salt composition were observed. In addition, variations of peak areas of these samples were within the range of the standard deviation observed for untreated samples. Therefore, we concluded that there are no significant differences in the DCL compositions for the evaluated ions, suggesting that the observed behavior is due to the ionic strength and not to specific interactions between the inorganic ions and the charged side chains of the pseudopeptides.

MCR-ALS results: When MCR-ALS was directly applied to the $\mathrm{HPLC}_{\mathrm{NaCl}}$ data set, the explained data variance was $99.14 \%$ with two components (or states of the library). The bar plot of the MCR-ALS states contributions (Figure $3 \mathrm{~A}$ ) showed that the first state (blue) must be related to the untreated condition, while the addition of $\mathrm{NaCl}$ produces the growth of a second salty state (red) that disturbs the equilibrium of the untreated sample. With just $0.5 \mathrm{M} \mathrm{NaCl}$, the salty state contribution is already $~ 50 \%$, and it increases linearly with the salt added. The batch differences observed in the PCA appear also here since the sample subset $b$ contains a $\sim 5-10 \%$ more contribution of state 2 than the subset $a$, pointing out again that these differences are not related to the amount of salt but to batch differences.
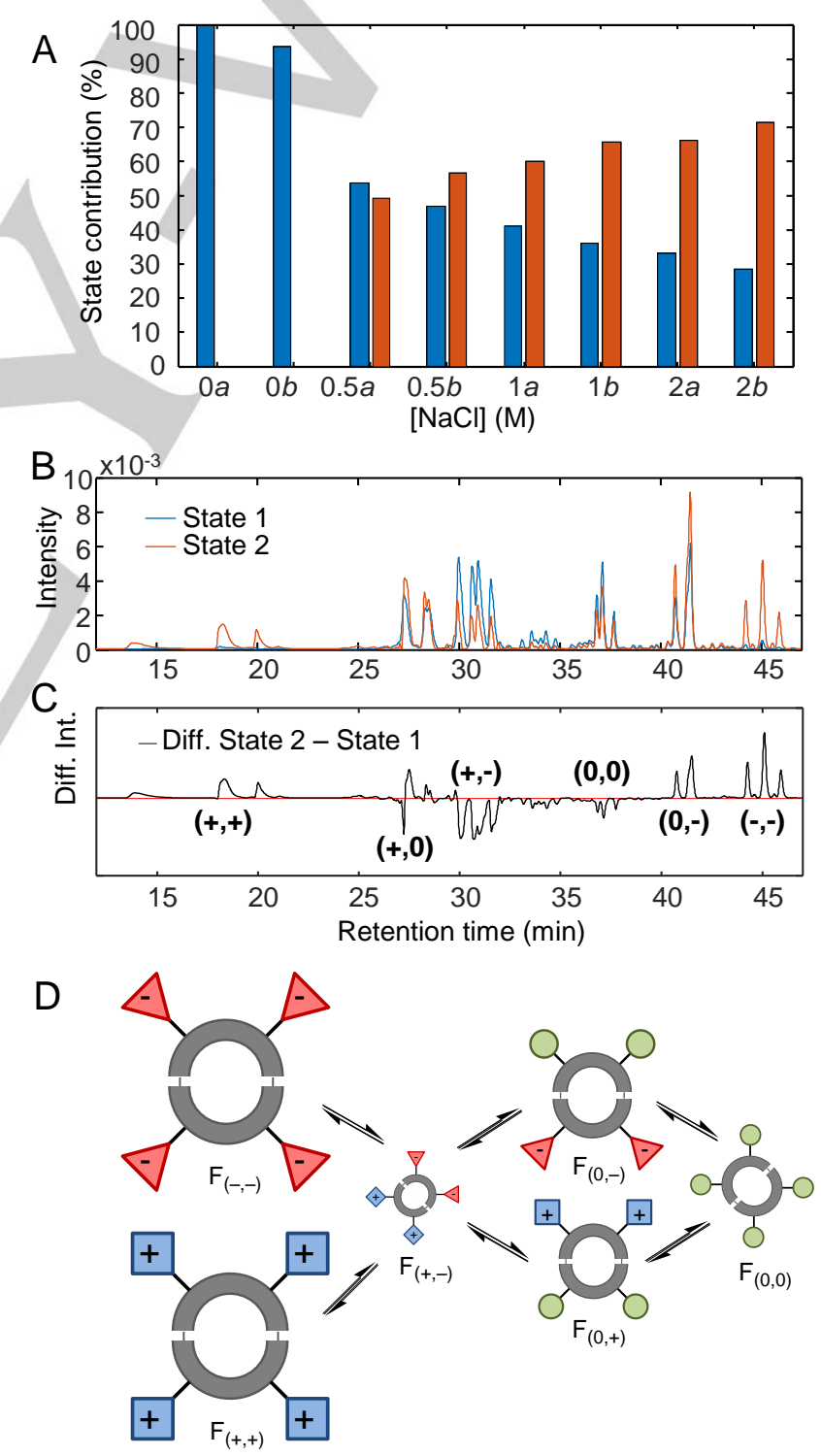
Figure 3. (A) Bar plots of the scores of the two states resolved by MCR-ALS analysis of the $\mathrm{HPLC}_{\mathrm{NaCl}}$ data set. (B) Loading profiles of the two states resolved by MCR-ALS. (C) Difference between the loading profiles of the two states resolved by MCR-ALS. (D) Schematic representation of the amplification of the families of macrocycles due to the salt effect (larger/smaller size symbols represent an increase/decrease of the concentration in the mixture).

MCR-ALS loadings of the library states are given in Figure 3B (Table S2) and its corresponding difference (state 2 - state 1) in Figure $3 \mathrm{C}$. They show that the main changes produced during the salt addition can be found in the $F_{+,+}, F_{-,-}$and $F_{+,-}$families. Thus, the $F_{+,+}$and $F_{-,-}$families were amplified by the salt addition, mainly at the expense of the $F_{+,-}$species. In quantitative terms,

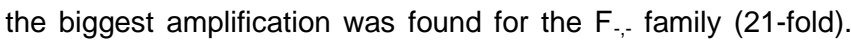
This rather large increase correlates with the more-in-depth thermodynamic analysis of this DCL upon the action of the same stimulus, which showed a more efficient salt adaptation of the tetra-anionic species. ${ }^{[1]}$ Very remarkably, the plot shown in Figure $3 \mathrm{C}$ is a good visual representation of the salt adaptive process itself, since it reflects the favored and disfavored species upon the action of the salt in a semi-quantitative way. This plot highlights the utility of MCR-ALS analysis for characterizing adaptive processes in dynamic chemical networks. The relative amplification of the families of species has been schematized in Figure 3D where the larger cartoons correspond to a larger effect of the salt.

\section{The combined effect of the ionic strength and the $\mathrm{pH}$ of the medium}

With the aim to increase the complexity of our dynamic system, we also considered a set of experiments where two interconnected stimuli are applied. This scenario is more challenging, since we expect to bisect the effect of interdependent triggers in a relatively complex dynamic network. To do that, we set up a series of experiments as a proof of concept. We decided to combine two mutually dependent environmental factors, such as the ionic strength and the $\mathrm{pH}$ of the medium. As shown in a previous section, the DCL is able to adapt its constitution to the presence of salt, due to the electrostatic shielding of attractive and repulsive interactions. This process must be influenced by the overall charge of the species within the library and, thus, a change in the $\mathrm{pH}$ leading to partial protonation/deprotonation of the corresponding side chains should have an impact on the system. Accordingly, we planned to generate and analyze the DCL at two different salt contents $(0$ and $1 \mathrm{M} \mathrm{NaCl})$ and two different $\mathrm{pH}$ values (4.8 and 6.5). We also tried the corresponding experiments at $\mathrm{pH} 9.0$, although the formation of over-oxidized species precluded their accurate analysis. Replicates were introduced to increase the pool of samples. The resulting DCL chromatographic data set obtained at different $\mathrm{pH}$ and salt content will be referred hereafter as the HPLC $\mathrm{pH}_{\mathrm{NaCl}}$ data set.

PCA results: As expected, the PCA of this set of samples showed that the final composition of the library depends on both the ionic strength and the $\mathrm{pH}$ (Figure $4 \mathrm{~A})$. The effect of $[\mathrm{NaCl}]$ is clearly defined by PC1 (71.60\% of the explained variance). The salt produces a major formation of tetra-charged dimers and a detriment of neutral dimers (Figure 4B). Besides, samples that contain $1 \mathrm{M} \mathrm{NaCl}$ (squares in Figure 4A) appear to be more clustered (regardless of the $\mathrm{pH}$ value) than the ones without $\mathrm{NaCl}$ (triangles in Figure 4A), implying that the effect of $\mathrm{pH}$ is lower at high salt concentration.
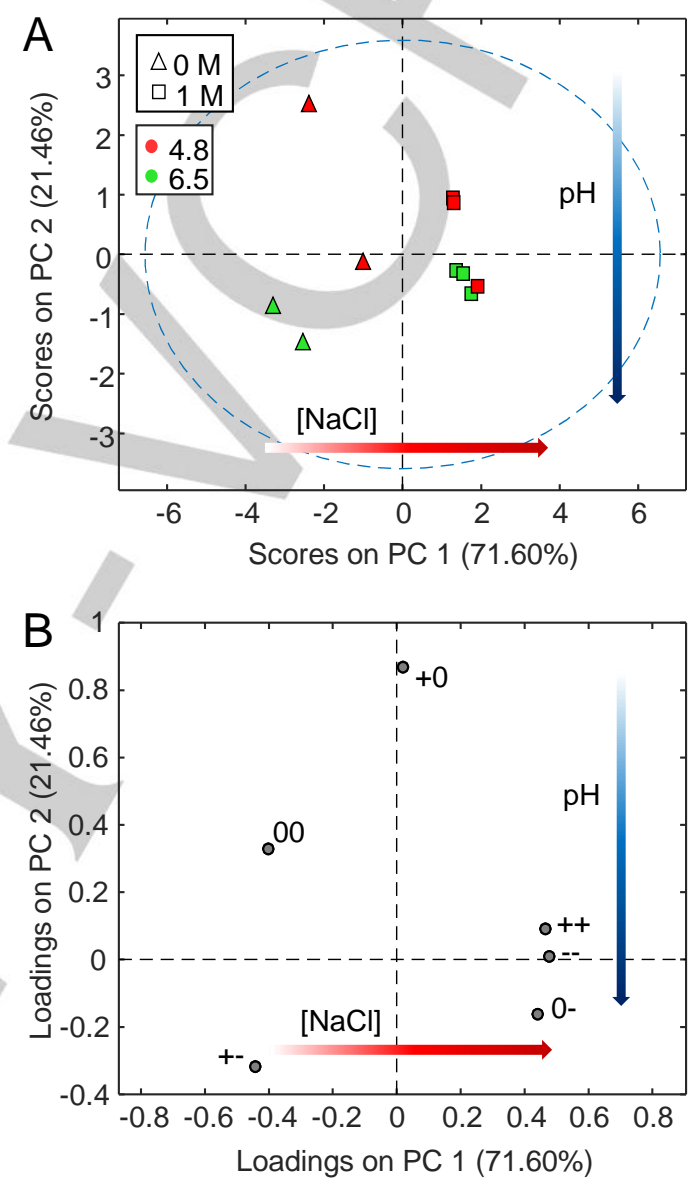

Figure 4. (A) Plot of the sample scores projected on the two first components obtained by PCA analysis of the DCL compositions obtained at different $\mathrm{pH}$ and salt content (HPLC $\mathrm{pH}_{\mathrm{NaCl}}$ data set). (B) Plot of the variable loadings projected on the two first components obtained by PCA analysis of the $\mathrm{HPLC}_{\mathrm{pH} \_\mathrm{NaCl}}$ data set.

The second principal component shown in Figure 4A (PC2, $21.46 \%$ of the explained variance) describes sample variations due to changes in $\mathrm{pH}$. More acidic samples (red symbols in Figure 4A) contain more $F_{+, 0}$ and $F_{0,0}$ species (Figure $4 B$ ), whereas neutral, more basic (green symbols in Figure $4 A$ ) samples contain more $F_{-, 0}$ and $F_{+,-}$species instead (Figure 4B). This trend can be also rationalized by electrostatic reasons, since the more acidic $\mathrm{pH}$ produces the partial protonation of the carboxylic side chains, reducing the electrostatic interactions of macrocycles containing either Glu or Asp amino acids.

MCR-ALS results: When MCR-ALS was applied to the HPLC $_{\mathrm{pH} \_\mathrm{NaCl}}$ data set (Figure 5 and Table S2), two states of the library were used and the explained data variance was $97.43 \%$. 
The main trend due to the salt effect was again the amplification of $F_{+,+}$and $F_{-,-}$macrocyclic dimers of the library and the decrease of the concentration of $F_{+,-}$species as previously observed in the PCA plots (Figure 4). Although the effect of the $\mathrm{pH}$ is not directly observed in the profiles of these two states, its contribution cannot be overlooked. In fact, as shown in Figure 5A, the amount of treated state was $\sim 25 \%$ without salt at $\mathrm{pH} 4.8$, but $\sim 15 \%$ without salt at $\mathrm{pH} 6.5$. This means that, in the absence of salt, the change in the $\mathrm{pH}$ from 6.5 to 4.8 produced a two-fold difference in the contribution of the treated state, which is quite remarkable. On the other hand, at $1 \mathrm{M} \mathrm{NaCl}$, the state contributions at different $\mathrm{pH}$ were practically identical ( 75\% contribution of the red state). Therefore, the effect of salt is much stronger than the $\mathrm{pH}$ effect, being the latter apparently negligible at $1 \mathrm{M} \mathrm{NaCl}$. This result implies that a highly saline medium efficiently shields the charged side chains, making the electrostatic interactions (either attractive in $F_{+,-}$or repulsive in $F_{+,+}$and $F_{-,-)}$less important. Under these circumstances, the protonation/deprotonation of the corresponding side chains would not affect the relative population of the different species and, thus, the $\mathrm{pH}$ of the medium would exert a very minor effect.
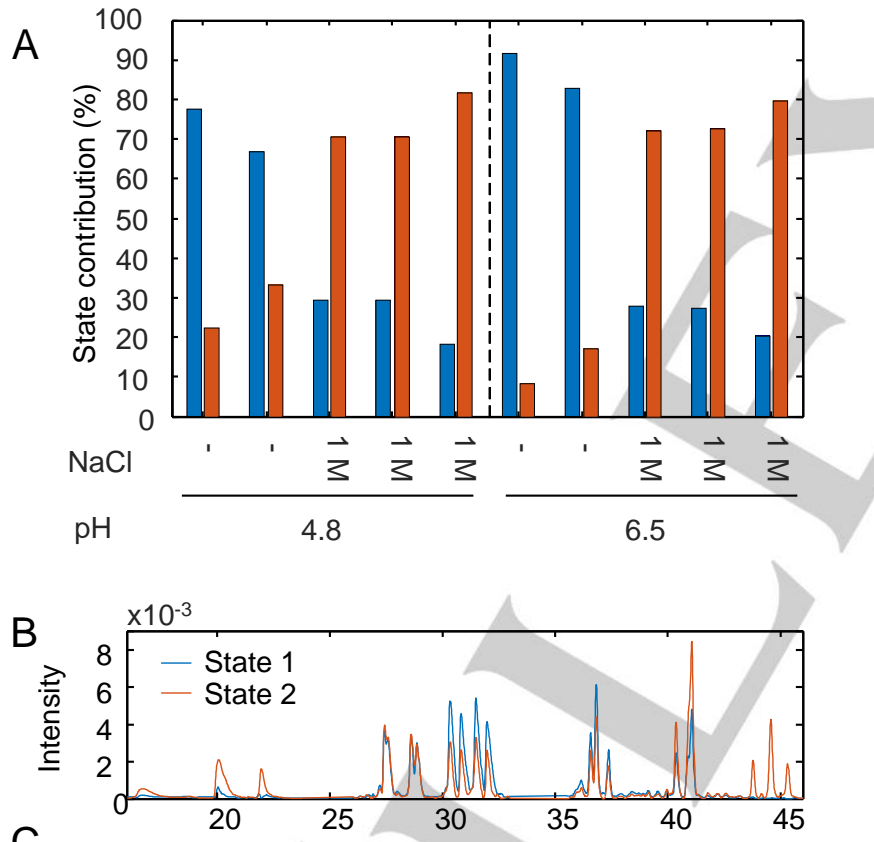

C

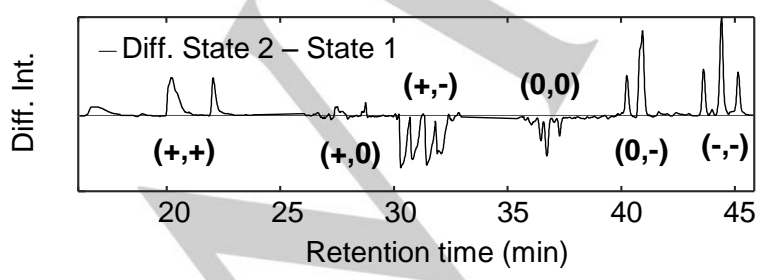

Figure 5. (A) Bar plots of the scores of the two states resolved by MCR-ALS

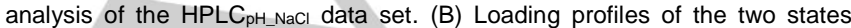
resolved by MCR-ALS. (C) Difference between the loading profiles of the two states resolved by MCR-ALS.
Summarizing, the combination of PCA and MCR-ALS analyses to the multi-stimuli responsive DCL rendered several important conclusions. First of all, chemometric data analysis allowed the grading of the two stimuli, clearly showing that the salt effect dominates over the $\mathrm{pH}$ effect. This can be rationalized by considering that the differences in the protonation degree are not so important at these two values of $\mathrm{pH}$. More interestingly, the clustering of the samples in the PCA and the careful interpretation of the MCR-ALS results showed that a highly saline medium compensates the effect of the different protonation degrees of the members of the library.

\section{Spermine polycation as a stimulus}

The addition of a template to a dynamic system can disturb the overall equilibrium composition if one or several members of the library interact with that template. This has been used to detect very efficient non-natural receptors for interesting guests. ${ }^{[11 a]}$ One of these biologically relevant guests is the biogenic polyamine spermine, which exists as polycationic species in aqueous solution at neutral or slightly acidic $\mathrm{pH}$. Actually, spermine has been already used to amplify members from a DCL of compounds bearing several carboxylate functions. ${ }^{[33]}$ More specifically, previous work in our group suggested that the homodimer formed by two BBs derived from Glu $\left(\mathbf{1 b}_{2}\right)$ is able to bind spermine in aqueous medium at $\mathrm{pH}$ 6.5. ${ }^{[28]}$ This macrocycle is a virtual member of the current DCL (along with two similar ones, like $\mathbf{1} \mathbf{a}_{2}$ and $\mathbf{1 a - 1} \mathbf{b}$ ), and we wondered if this interaction could be also manifested in the composition of a more complex library.

To study the effect of spermine in our chemical system, we generated the $\mathrm{DCL}$ at the same conditions $(0.5 \mathrm{mM}$ in each $\mathrm{BB}$, $40 \mathrm{mM}$ bis-Tris buffer, $25 \%$ DMSO in water, $\mathrm{pH} \mathrm{6.5)} \mathrm{but} \mathrm{in} \mathrm{the}$ presence of increasing amounts of spermine from 0 to $100 \mathrm{mM}$ (Figure 6). The stock solution of the spermine used for this titration experiment was pre-adjusted to $\mathrm{pH} 6.5$ with $\mathrm{HCl}$ and thus, it contains the protonated form of the polyamine and the corresponding chloride counterions. We anticipated that the effect of the polyamine should be higher than the simple effect of the ionic strength and, accordingly, the experiments were carried out starting with low concentrations of the polyamine. 


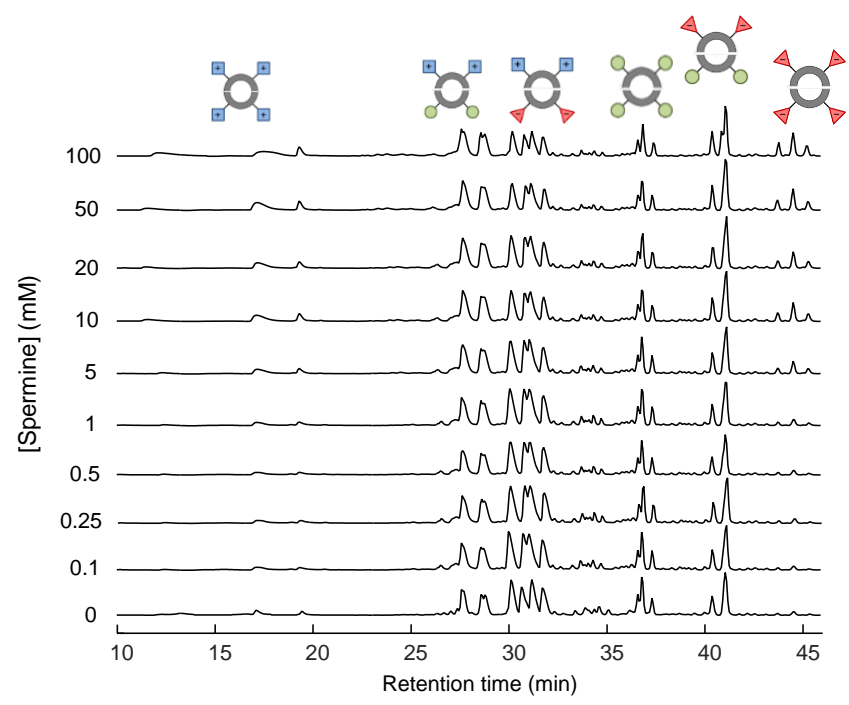

Figure 6. Stacked HPLC traces of the DCL of the pseudopeptidic macrocycles at increasing concentrations of spermine ( $\left.\mathrm{HPLC}_{\text {spermine }}\right)$.

PCA results: In general, the response of the DCL to increasing concentrations of this more elaborated polyelectrolyte was similar to that observed with increasing amounts of salt (Figure 6 ): the presence of spermine produced an increase of fully charged macrocyclic dimers formation $\left(\mathrm{F}_{+,+}\right.$and $\left.\mathrm{F}_{-,-}\right)$. However, as observed in the PCA scores and loadings plots of Figure 7 , this response is not linearly changing as in the case of increasing salt concentrations. In the PCA analysis of Figure 7, $98.06 \%$ of the data variance was explained using two principal components. The first principal component (PC1, 88.43\% of the explained variance) shows a pattern similar to the $\mathrm{NaCl}$ case. In contrast, PC2 (9.63\% of the explained variance) is associated to a minor underlying adaptive pattern, which has its maximum contribution at $5 \mathrm{mM}$ spermine, but decreases when more spermine is added (Figure 7A). The fact that loadings distribution on Figure 7B was different to the ones at increasing $\mathrm{NaCl}$ concentrations (Figure 2B) points out again that the charged dimers formation are not equally favored in the presence of spermine as in presence of the $\mathrm{NaCl}$ salt. A reasonable explanation to this behavior should be due to specific supramolecular interactions (either repulsive or attractive) of some of the members of the library with the polycationic spermine molecule. Thus, in this case, not only the ionic strength was playing an important role but also the presence of the spermine structure as a template.

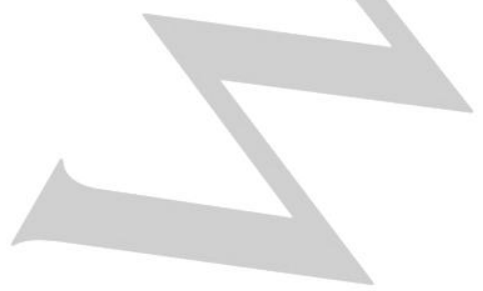

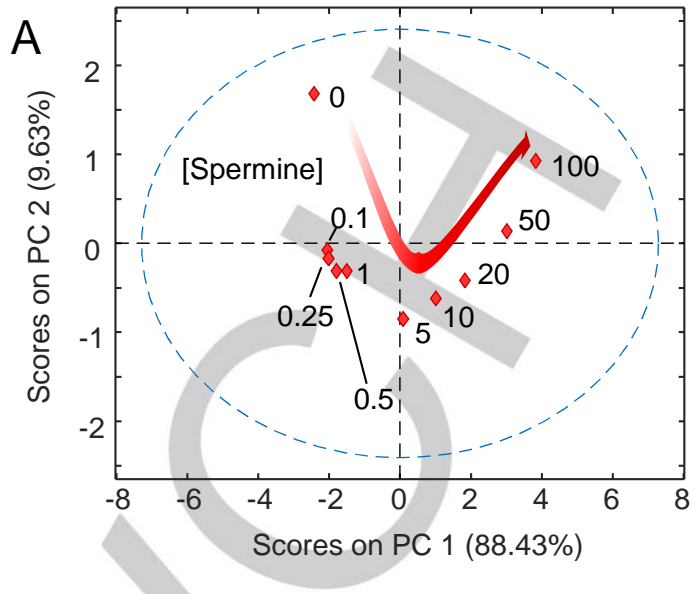

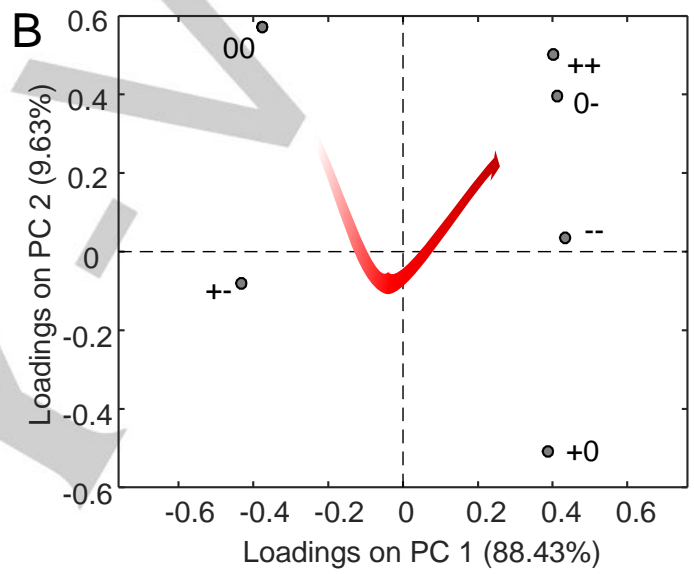

Figure 7. Plot of the sample scores (A) and of the loadings $(B)$ projected on

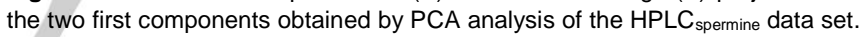

MCR-ALS results: When MCR-ALS was applied to the same dataset to investigate the influence of increasing spermine concentrations on the DCL distribution ( HPLC $\left._{\text {spermine }}\right)$, two components (states) were used to have an explained data variance of $97.18 \%$ (Figure S2). As in the PCA score plot analysis (Figure 7A), a two-stage gradual change process (from 0 to $5 \mathrm{mM}$, and from 5 to $100 \mathrm{mM}$ ) was observed depending on spermine concentration in the MCR-ALS analysis (Figure S2A). Despite this different pattern in the contribution of the two states, the underlying tendencies extracted from the HPLC profiles (Figures S2B) were rather similar to the ones discussed for the previously investigated $\mathrm{NaCl}$ effect. Thus, the presence of spermine amplifies the presence of tetra-charged species at the expense of the neutral zwitterionic ones. This could be explained by the higher electrostatic interactions of spermine with the macrocycles of the $F_{-,-}$family, with the concomitant rearrangement of the others members of the mixture. However, the observed non-linear trend was somehow intriguing and we deemed to get additional information on this regard.

Separation of ionic strength and supramolecular interaction effects with MCR-ALS: The addition of spermine to the DCL 
can produce two effects on the library. On one hand, the putative supramolecular interactions with some of the pseudopeptidic macrocycles would stabilize the members that interact with the spermine more efficiently. On the other hand, the increase of the concentration of this substrate would concomitantly increase the ionic strength of the medium, since spermine is a polycationic molecule (together with the corresponding chloride anions). As the library of pseudopeptidic macrocycles is responsive to the ionic strength, the observed adaptation of the mixture must be due to the combined response to the two stimuli. However, these two dependent stimuli are not orthogonal, but linearly correlated and thus experimentally inseparable. Accordingly, their separation was investigated by chemometric approaches. In order to do that, the two sets of samples previously analyzed individually $\left(\mathrm{HPLC}_{\mathrm{NaCl}}\right.$ and HPLC $_{\text {spermine}}$ ) were simultaneously analyzed by MCR-ALS. This system was resolved using now three components as shown in Figure 8 (Table S2). During the MCR-ALS analysis, samples at different salt concentrations (without spermine) were forced to be resolved with only two components (states of the $\mathrm{DCL}$ ): one related to non-salt, state 1 in blue, and one related to high salt concentrations, state 2 in red. The third state was included only for the analysis of the HPLC spermine $_{\text {dataset. With this constraint }}$ in the $\mathrm{HPLC}_{\mathrm{NaCl}}$ subset, and by allowing the states 1 and 2 to be in common with the HPLC spermine dataset, the exclusive effect produced by spermine can be better distinguished from the effect of just increasing the ionic strength. This effect is described by the state that is specific for the HPLC spermine dataset (component 3 in green). The explained variance for this combined data set was $97.53 \%$, which was reasonably good compared to those given above for the two data sets analyzed individually

As shown in Figure 8A, the evolution of samples containing increasing amounts of spermine was satisfactorily resolved when three states were used. Two of them are similar to those obtained when $\mathrm{HPLC}_{\mathrm{NaCl}}$ was analyzed (one related to no salt, in blue, and another dominant at higher ionic strength values, in red). A third state was distinguished only for spermine samples (green in Figure $8 \mathrm{~A}$ ) which already appeared at very low concentrations of spermine $(0.1 \mathrm{mM})$. In this case the state associated to the increase of the ionic strength did not appear until the concentration of spermine was $5 \mathrm{mM}$. First of all, this would explain the non-linear behavior observed in the PCA score plot of Figure 7A, where the sample containing $5 \mathrm{mM}$ spermine showed the maximum contribution to PC2. Secondly, the specific contribution of the third state (green) in the range 0.1-1.0 mM spermine is not related to the increase of the ionic strength. Besides, since this is effective at such low concentrations, it should correspond to a relatively strong supramolecular specific interaction. Thus, we assigned the green state to the binding of spermine to some of the members of the library. For samples containing spermine concentrations higher than $5 \mathrm{mM}$, both the specific binding (green) state and the non-specific ionic strength (red) state are participating in the $\mathrm{DCL}$ composition, being the relative contribution of the red one more important at higher spermine concentrations, as expected.

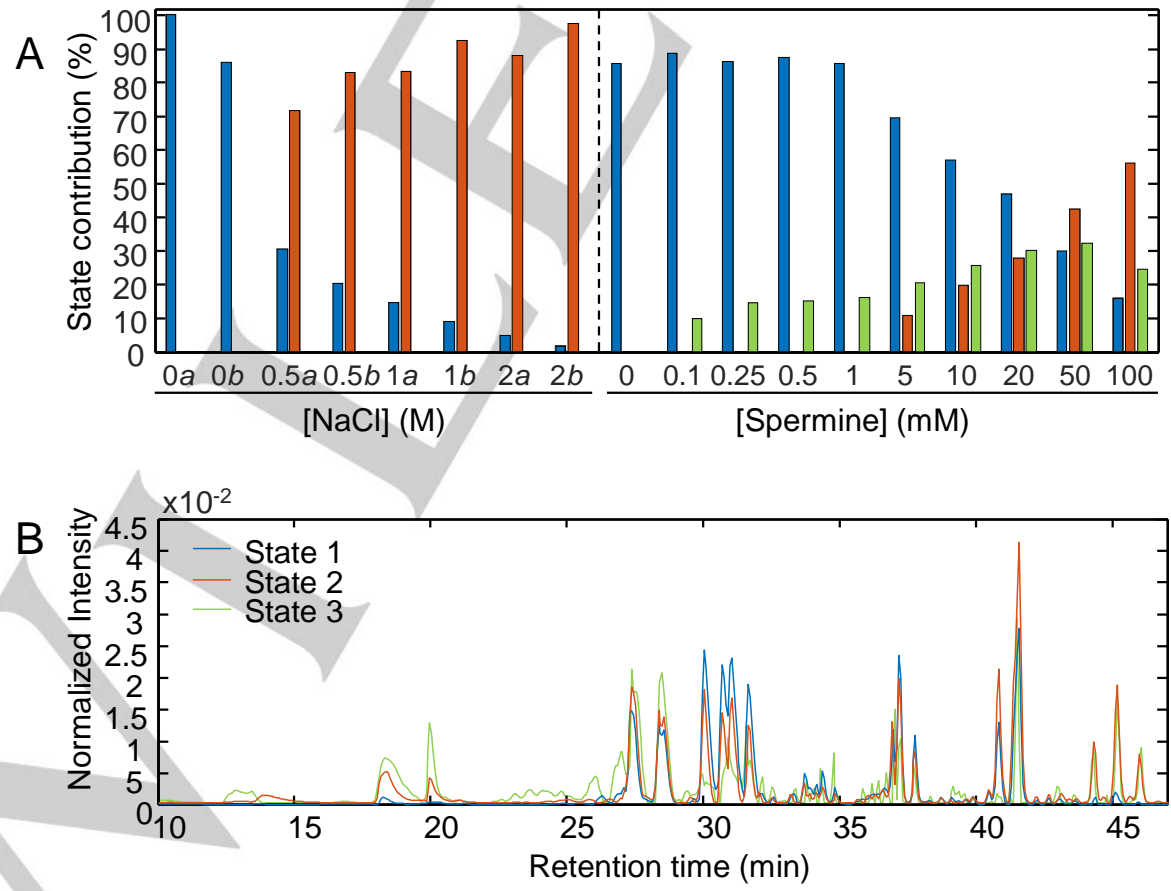

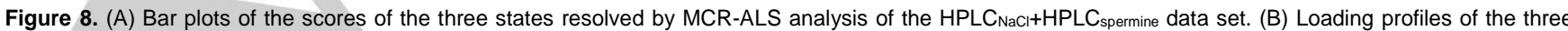
components resolved by MCR-ALS analysis of the HPLC $\mathrm{NaCl}_{\mathrm{H}}+\mathrm{HPC}_{\text {spermine }}$ data set. 
We also aimed to get more precise information about the adaptive process by using the plots of the difference between states at chromatographic resolution. The differences between state 2 (high ionic strength $\mathrm{DCL}$ ) and state 1 (untreated DCL) are depicted in Figure 9A (black trace). The evolution of the members of the library is very similar to the one observed for the MCR-ALS analysis of the exclusive effect of the salt (Figure 3C) as clearly shown by overlaying the corresponding trace (blue trace in Figure 9A).
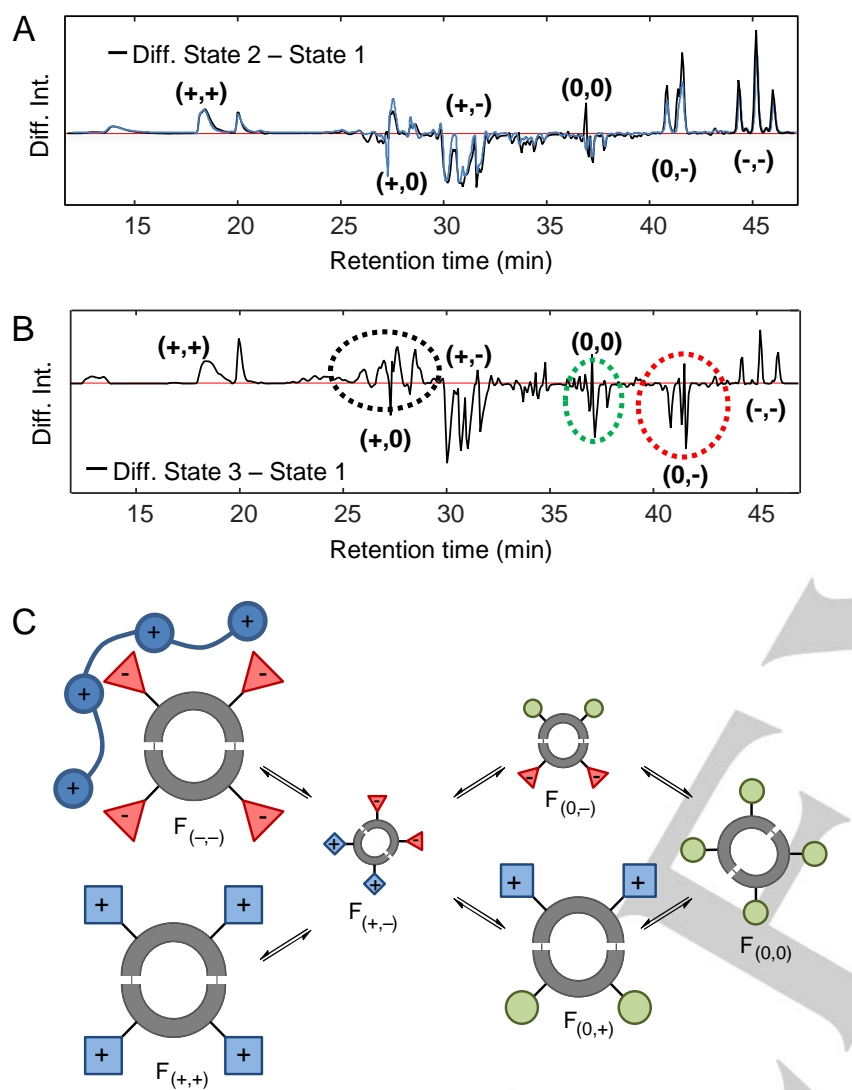

Figure 9. (A) Differences of state 2 (high ionic strength $D C L$ ) minus state 1 (untreated $\mathrm{DCL}$ ) for the $\mathrm{HPLC}_{\mathrm{NaCl}}+\mathrm{HPLC}_{\text {spermine combined dataset (black }}$ trace) and the HPLC $\mathrm{NaCl}$ dataset (blue trace). (B) Differences of state 3 (spermine bound $D C L$ ) minus state 1 (untreated $D C L$ ) for the $\mathrm{HPLC}_{\mathrm{NaCl}}+$ HPLC spermine combined dataset. The main differences with $(A)$ are highlighted with dashed regions. (C) Cartoon representation of the effect of the spermine polycation binding on the DCL (larger/smaller size symbols represent an increase/decrease of the concentration in the mixture).

This plot supports that the effect of the ionic strength has been efficiently resolved in both analysis. The differences between state 3 (spermine bound DCL) and state 1 (untreated $D C L$ ) would show the evolution of the dynamic system exclusively due to the supramolecular interactions with spermine (Figure 9B). Remarkably, the trends are very different to the ones observed due to the increase of the ionic strength. Thus the most striking change is observed in the decrease of $F_{0,0}$ and, specially, $F_{0,-}$ families. This can be solely explained by the selective amplification of the $F_{-,-}$family, which sequesters the anionic building blocks from the mixture. Thus, all the positively charged species $F_{+,+}$and $F_{0,+}$ are just opportunistically amplified in this case. The unsymmetrical behavior of the evolution of $F_{0,+}$ (amplified) and $F_{0,-}$ (reduced) further supports that the spermine specifically amplifies the $F_{-,-}$species, most likely by the binding through electrostatic and $\mathrm{H}$-bonding interactions. This scenario has been schematically depicted in the cartoons shown in Figure $9 \mathrm{C}$.

Overall, this data analysis shows that the effect of increasing concentrations of spermine is more elaborated than the simple increase of the ionic strength. Thus, we have identified the supramolecular interactions of spermine with the homo- and heterodimers derived from Asp and Glu, which are tetra-anionic at this $\mathrm{pH}$ and interact more strongly with spermine.

\section{Conclusions}

Multivariate data analysis approaches are commonly used for analyzing complex data systems in several areas of scientific research, such as environmental science, analytical chemistry and ecology among others. Here we have demonstrated the power of these chemometric data analysis methods for unraveling compositional changes in relatively complex dynamic combinatorial libraries. As a proof of concept, we have used a dynamic mixture of macrocyclic pseudopeptides bearing differently charged side chains and thus, prone to respond to ionic stimuli. The effects of the ionic strength have been clearly characterized both qualitatively (PCA) and semi-quantitatively (MCR-ALS), showing trends in very good agreement with the more time-consuming thermodynamic characterization, previously performed by dynamic deconvolution. The more challenging analysis of the evolution of the complex system upon the action of two interdependent stimuli has been also unraveled with the chemometric methods, bisecting the influence of ionic strength and $\mathrm{pH}$ on the library composition. Additionally, both PCA and MCR-ALS reflected the differences in the dynamic adaptation produced by a cationic polyelectrolyte such as spermine, suggesting that there are specific supramolecular interactions between the polyamine and some members of the library. The effect of this binding can be cleared from the concomitant increase of the ionic strength also produced by the spermine polycation by using the MCR-ALS analysis. Overall, we have shown that these chemometric analyses are extremely useful for the high throughput screening of many different stimuli in relatively complex chemical systems. They show to be especially useful when several stimuli are applied either in a linearly independent/orthogonal way $\mathrm{pH}$ and ionic strength) or in a linearly dependent fashion (binding to a charged substrate and ionic strength). Moreover, although we applied our study to a dynamic mixture operating under equilibrium, we envision that similar protocols could be used for out-of-equilibrium systems, which can be more interesting in terms of Systems Chemistry studies and toward future applications of molecular networks. One of these applications would be within the field of molecular sensors based on dynamic 
networks, where the chemometric tools will be specially powerfull not only for pattern recognition but also for the understanding of the systems at the molecular level.

\section{Experimental Section}

The pseudopeptidic building blocks 1a-f were prepared as previously reported. ${ }^{[21,28]}$ The corresponding dynamic combinatorial libraries were generated following slight variations from the described procedure, ${ }^{[21]}$ adapting the conditions to the implementation of the given stimulus (see below).

\section{Generation of the DCLs upon the action of the different stimuli}

The DCLs were prepared by dilution of $12 \mathrm{mM}$ individual stocks of 1a-f prepared in DMSO. Four DCLs studies were performed: the study of the salt effect (study 1), the study of the influence of different inorganic salts (study 2), the study of the influence of $\mathrm{pH} \&$ salt (study 3), and the study of the influence of spermine concentration (study 4). For these studies, the reaction mixtures were prepared by dilution of a stock mixture of the corresponding BBs, ensuring no differences in concentration between the libraries of the same batch. The DCLs of the complex system generated from the mixture of 1a-f were performed at $0.5 \mathrm{mM}$ of each BB in a 40 $\mathrm{mM} \mathrm{BIS-Tris} \mathrm{buffer} \mathrm{(pH} \mathrm{6.5)} \mathrm{with} 25 \%$ DMSO in the presence of $0.0-2.0$ $\mathrm{M} \mathrm{NaCl}$ (study 1). Likewise, solutions for the different inorganic salts were prepared in BIS-Tris buffer ( $\mathrm{pH} \mathrm{6.5)}$ to achieve a final ionic strength equivalent to $1.0 \mathrm{M} \mathrm{NaCl}$. Thus, $0.33 \mathrm{M}$ final concentrations of $\mathrm{CaCl}_{2}$, $\mathrm{MgCl}_{2}$ or $\mathrm{Na}_{2} \mathrm{SO}_{4}$ were used and $0.25 \mathrm{M} \mathrm{MgSO}_{4}$ was employed (study 2). Reactions at pH 4.8 were carried out using formate buffer (study 3). For reactions containing spermine a stock solution of spermine $(133 \mathrm{mM})$ in BIS-Tris buffer was adjusted to $\mathrm{pH} 6.5$ with $4 \mathrm{M} \mathrm{HCl}$ (study 4). Dilutions from the stock solutions were employed to achieve the final concentrations described.

\section{HPLC analysis}

Once the oxidation of the free thiols was complete (typically after 24 hours at room temperature) the reaction mixtures were analyzed by HPLC. The HPLC samples were prepared by adding $45 \mu \mathrm{L}$ of the reaction mixture to $75 \mu \mathrm{L}$ of a solution of $89 \% \mathrm{H}_{2} \mathrm{O}, 10 \% \mathrm{CH}_{3} \mathrm{CN}$ and $1 \%$ TFA. For the chromatographic analysis of the DCLs we utilized a reversed-phase kromaphase $\mathrm{C}_{18}(25 \times 0.46 \mathrm{~cm}, 5 \mu \mathrm{m})$ column. $\left(\mathrm{CH}_{3} \mathrm{CN}+\right.$ $20 \mathrm{mM} \mathrm{HCOOH}$ and $\mathrm{H}_{2} \mathrm{O}+20 \mathrm{mM} \mathrm{HCOOH}$ ) mixtures at $1 \mathrm{~mL} / \mathrm{min}$ were used as mobile phase and the monitoring wavelength was set at $254 \mathrm{~nm}$. The gradient program used was: 10 min at $2 \% \mathrm{CH}_{3} \mathrm{CN}$ in $\mathrm{H}_{2} \mathrm{O}$, then linear gradient from $2 \%$ to $40 \% \mathrm{CH}_{3} \mathrm{CN}$ over 62 min (both $\mathrm{CH}_{3} \mathrm{CN}$ and $\mathrm{H}_{2} \mathrm{O}$ containing $20 \mathrm{mM} \mathrm{HCOOH}$ ).

\section{Chemometric analysis}

Data pretreatment: First, elution profiles were imported to Matlab environment (The Mathworks Inc. Natick, MA, USA). For every sample, one vector of absorbance intensities measured at the wavelength of 254 $\mathrm{nm}$ with as many variables as measured elution times was generated. In order to create a matrix of absorbance intensity vectors, elution times were interpolated. A I-by-J matrix (42 samples and 1,100 elution times) was generated. Regions where macrocycles did not elute were excluded from the analysis. Baseline correction was performed using the Automatic Weighted Least Squares algorithm from the PLS toolbox 8.0.2 (Eigenvector Research Inc., Wenatchee, WA, USA). Chromatograms from samples of study 1 (salt effect) and of study 4 (spermine) were aligned at the same time axis with the icoshift algorithm ${ }^{[34]}$. Samples of study 2 (inorganic salts) and study 3 (the combined effect of $\mathrm{pH}$ and salt) were aligned separately using the same algorithm. Thus, three resulting data matrices (one per alignment) were obtained. Each chromatogram was normalized to its total area. The three resulting matrices contain as many rows as samples, and $\mathrm{J}=600$ measured elution times per sample (in columns). In addition, for each data matrix, integration of the individual peak areas relative to the 6 possible families of macrocyclic dimers $\left(F_{+,+}\right.$, $F_{+, 0}, F_{0,0}, F_{+,-,}, F_{0,-}$ and $F_{-,-)}$was performed by the sum of the peak intensities that define each given family of dimers. With the resulting integrals, peak area data matrices with as many rows as samples, and of $\mathrm{J}=6$ variables (one variable per peak integral or family, in columns) were created.

Principal Component Analysis: Principal Component Analysis (PCA) ${ }^{[30]}$ was applied to the auto-scaled peak area data matrices. The goal of this analysis was to provide an initial qualitative observation of the reassembling patterns (composition changes) of the differently charged macrocycle dimers of the DCL and to determine which macrocycle families were more influenced by the triggering factors (salinity, ionic strength, ionic charge, spermine concentration, and $\mathrm{pH}$ ). PCA produces a reduced number of new orthogonal variables (or principal components, PCs), built from linear combination of the original variables, which keep most of the original data variance. This compression is achieved by means of a bilinear decomposition of the raw data into two factor matrices: the matrix of scores and the matrix of loadings. The scores give information about how the data samples are distributed in the new orthogonal space defined by the PCs. By visualizing the sample scores using scatter plots it is possible to elucidate the similarities among samples (i.e. closer samples will have similar scores, and hence, similar variances within the studied set of samples). The loadings give information about what is the contribution of the original variables in the new orthogonal space defined by the PCs. PCA was performed using the PLS toolbox 8.0.2 under Matlab environment.

Multivariate Curve Resolution by Alternating Least Squares: In order to resolve the main composition changes and patterns of the $\mathrm{DCL}$ triggered by the changes in different experimental conditions (ionic strength, salt, $\mathrm{pH}$, spermine, etc.), the chromatographic data sets obtained in these experiments were examined by the Multivariate Curve Resolution by Alternating Least Squares (MCR-ALS) method ${ }^{[31 b]}$ using the MCR-ALS GUI 2.0[35] under Matlab environment. MCR-ALS is a chemometric data analysis method which decomposes a given data matrix in the product of two factor matrices using the following bilinear model (Eqn.1):

\section{$\mathrm{X}=\mathrm{GF}^{\mathrm{T}}+\mathrm{E}$}

(Eqn.1)

Here the data matrix $\mathbf{X}(\mathrm{I}, \mathrm{J})$ contains the set of I chromatograms with $\mathrm{J}$ elution times obtained in the analysis of the DCL mixtures at the different conditions (ionic strength, salt, $\mathrm{pH}$, spermine). In this particular case, MCR-ALS gives two factor matrices, $F^{\top}(N, J)$ and $G(I, N)$. The chromatographic profiles (row vectors) in $\mathbf{F}^{\top}$ reveal the changes in the composition of the different library components associated to the stimuli (states of the library), since every peak in $\mathbf{F}^{\mathbf{T}}$ can be related to one member of the library. On the other hand, $\mathbf{G}$ shows the relative contribution of these changes in the chromatographic profiles for every sample. $\mathrm{N}$ represents the number of components (or states) used in the decomposition generated in the MCR-ALS analysis. $\mathrm{N}$ can be initially

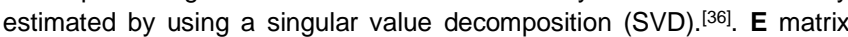
(size I, J) contains the residual information not explained by the model using the $\mathbf{N}$ considered components. The estimation of $\mathbf{G}$ and $\mathbf{F}^{\mathbf{T}}$ factor matrices was performed by means of an alternating least squares optimization (ALS) under constraints. In this work, for the analysis of 
individual datasets corresponding to the investigation of the changes produced by one of the factors (ionic strength, $\mathrm{pH}$, spermine), nonnegativity constraints were applied on both $\mathbf{G}$ and $\mathbf{F}^{\mathbf{T}}$ factor matrices as well as a closure constraint on $\mathbf{G}$ matrix that fixes a constant mass balance $^{[31 a]}$ in the sum of the DCL contributions on every sample. On the other hand, for the simultaneous MCR-ALS analysis of the combined $\mathrm{HPLC}_{\mathrm{NaCl}}+\mathrm{HPLC}_{\text {spermine }}$ data set, non-negativity constraints on both $\mathbf{G}$ and $\mathbf{F}^{\mathbf{T}}$ factor matrices and a selectivity constraint were imposed. This selectivity constraint was used to separate the contribution related to the presence of spermine (which is not present in samples treated only with $\mathrm{NaCl}$ ). Thus, the combined dataset was set to be modelled with three components, where two of them were common for the two subsets, and the third one related to the possible spermine supramolecular interaction

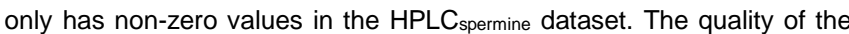
MCR-ALS model was measured by evaluating by the percent of explained variance $\left(R^{2}\right){ }^{[35]}$

\section{Acknowledgements}

Financial Support from MINECO/FEDER (CTQ2015-70117-R and CTQ2015-66254 projects), Generalitat de Catalunya (AGAUR, 2014 SGR 231) and European Union (COST CM1304) are gratefully acknowledged.

Keywords: systems chemistry $\cdot$ dynamic combinatorial libraries - macrocycles $\cdot$ multivariate data analysis $\bullet$ pseudopeptides

[1] (a) A. Vespignani, Nat. Phys. 2012, 8, 32-39; (b) J.-M. Lehn, Angew. Chem. Int. Ed. 2013, 52, 2836-2850; Angew. Chem. 2013, 125, 2906 2921.

[2] N. Pearcy, N. Chuzhanova, J. J. Crofts, J. Theor. Biol. 2016, 406, 99 104.

[3] F. Schweitzer, G. Fagiolo, D. Sornette, F. Vega-Redondo, A. Vespignani, D. R. White, Science 2009, 325, 422-425.

[4] (a) J. M. Montoya, S. L. Pimm, R. V. Solé, Nature 2006, 442, 259-264; (b) S. Azaele, S. Pigolotti, J. R. Banavar, A. Maritan, Nature 2006, 444 926-928.

[5] S. P. Borgatti, A. Mehra, D. J. Brass, G. Labianca, Science 2009, 323 , 892-895.

[6] I. Alfonso, Chem. Commun. 2016, 52, 239-250.

[7] S. N. Semenov, L. J. Kraft, A. Ainla, M. Zhao, M. Baghbanzadeh, V. E. Campbell, K. Kang, J. M. Fox, G. M. Whitesides, Nature 2016, 537 656-660.

[8] (a) R. F. Ludlow, S. Otto, Chem. Soc. Rev. 2008, 37, 101-108; (b) R. A. R. Hunt, S. Otto, Chem. Commun. 2011, 47, 847-858.

[9] K. Ruiz-Mirazo, C. Briones, A. de la Escosura, Chem. Rev. 2013, 114, 285-366.

[10] (a) S. Otto, Acc. Chem. Res. 2012, 45, 2200-2210; (b) E. Mattia, S. Otto, Nat. Nanotechnol. 2015, 10, 111-119; (c) J.-M. Lehn, Angew. Chem. Int. Ed. 2015, 54, 3276-3289; Angew. Chem. 2015, 127, 3326 3340; (d) G. M. Whitesides, R. F. Ismagilov, Science 1999, 284, 89-92.

[11] (a) P. T. Corbett, J. Leclaire, L. Vial, K. R. West, J.-L. Wietor, J. K. M. Sanders, S. Otto, Chem. Rev. 2006, 106, 3652-3711; (b) J. Li, P Nowak, S. Otto, J. Am. Chem. Soc. 2013, 135, 9222-9239; (c) F. B. L. Cougnon, J. K. M. Sanders, Acc. Chem. Res. 2011, 45, 2211-2221; (d) J. N. Reek, S. Otto, Dynamic combinatorial chemistry, John Wiley \& Sons, 2010; (e) Y. Jin, C. Yu, R. J. Denman, W. Zhang, Chem. Soc Rev. 2013, 42, 6634-6654.

[12] F. Biedermann, H.-J. Schneider, Chem. Rev. 2016, 116, 5216-5300.
[13] (a) I. Huc, J.-M. Lehn, Proc. Natl. Acad. Sci. U.S.A. 1997, 94, 2106 2110; (b) J.-M. Lehn, Chem. Eur. J. 1999, 5, 2455-2463; (c) J.-M. Lehn Chem. Soc. Rev. 2007, 36, 151-160.

[14] (a) F. B. L. Cougnon, N. A. Jenkins, G. D. Pantoş, J. K. M. Sanders Angew. Chem. Int. Ed. 2012, 51, 1443-1447; Angew. Chem. 2012, 124 , 1472-1476; (b) F. B. L. Cougnon, H. Y. Au-Yeung, G. D. Pantoş, J. K M. Sanders, J. Am. Chem. Soc. 2011, 133, 3198-3207; (c) N Ponnuswamy, F. B. L. Cougnon, J. M. Clough, G. D. Pantoş, J. K. M Sanders, Science 2012, 338, 783-785.

[15] (a) P. T. Corbett, S. Otto, J. K. M. Sanders, Chem. Eur. J. 2004, 10 3139-3143; (b) R. T. S. Lam, A. Belenguer, S. L. Roberts, C. Naumann, T. Jarrosson, S. Otto, J. K. M. Sanders, Science 2005, 308, 667-669.

[16] (a) B. Brisig, J. K. M. Sanders, S. Otto, Angew. Chem. Int. Ed. 2003, 42, 1270-1273; Angew. Chem. 2003, 115, 1308-1311; (b) L. Vial, J. K. M. Sanders, S. Otto, New J. Chem. 2005, 29, 1001-1003.

[17] E. Moulin, G. Cormos, N. Giuseppone, Chem. Soc. Rev. 2012, 41, 1031-1049.

[18] (a) J. J. P. Peyralans, S. Otto, Curr. Opin. Chem. Biol. 2009, 13, 705 713; (b) J. R. Nitschke, Nature 2009, 462, 736-738.

[19] (a) J. Atcher, A. Moure, I. Alfonso, Chem. Commun. 2013, 49, 487-489; (b) J. M. A. Carnall, C. A. Waudby, A. M. Belenguer, M. C. A. Stuart, J. J.-P. Peyralans, S. Otto, Science 2010, 327, 1502-1506; (c) J. W Sadownik, E. Mattia, P. Nowak, S. Otto, Nat. Chem. 2016, 8, 264-269.

[20] (a) J. Solà, M. Lafuente, J. Atcher, I. Alfonso, Chem. Commun. 2014 50, 4564-4566; (b) M. Lafuente, J. Atcher, J. Solà, I. Alfonso, Chem. Eur. J. 2015, 21, 17002-17009.

[21] J. Atcher, A. Moure, J. Bujons, I. Alfonso, Chem. Eur. J. 2015, 21 , 6869-6878.

[22] T. Bunyapaiboonsri, O. Ramström, S. Lohmann, J.-M. Lehn, L. Peng, M. Goeldner, ChemBioChem 2001, 2, 438-444.

[23] (a) For general information on chemometrics, see: Comprehensive Chemometrics: Chemical and Biochemical Data Analysis Ed. S. D. Brown, R. Tauler, B. Walczak, Elsevier, Amsterdam, 2009. (b) For a general discussion on different approaches to analyze DCLs, see: M. C. Misuraca, E. Moulin, Y. Ruff, N. Giuseppone, New J. Chem. 2014, 38 3336-3349.

[24] (a) L. You, D. Zha, E. V. Anslyn, Chem. Rev. 2015, 115, 7840-7892. (b) D. Zamora-Olivares, T. S. Kaoud, K. N. Dalby, E. V. Anslyn, J. Am Chem. Soc. 2013, 135, 14814-14820. (c) J. Zhang, L. You, E. V Anslyn, X. Qian, Chem. Eur. J. 2012, 18, 1102-1110. (d) B. E. Collins, E. V, Anslyn, Chem. Eur. J. 2007, 13, 4700-4708.

[25] (a) Z. Kostereli, R. Scopelliti, K. Severin, Chem. Sci. 2014, 5, 2456 2460; (b) B. Lee, S. Chen, C. Heinis, R. Scopelliti, K. Severin, Org Lett. 2013, 15, 3456-3459. (c) K. Severin, Curr. Opin. Chem. Biol. 2010, 14 737-742. (d) S. Rochat, J. Gao, X. Qian, F. Zaubitzer, K. Severin Chem. Eur. J. 2010, 16, 104-113. (e) A. Buryak, A. Pozdnoukhov, K Severin, Chem Commun. 2007, 2366-2368.

[26] (a) T. Takeuchi, S. Matile, Chem. Commun. 2013, 49, 19-29. (b) J. Montenegro, S. Matile, Chem. Asian J. 2011, 6, 681-689. (c) T. Takeuchi, J. Montenegro, A. Hennig, S. Matile, Chem. Sci. 2011, 2 , 303-307 (d) J. Montenegro, P. Bonvin, T. Takeuchi, S. Matile, Chem. Eur. J. 2010, 16, 14159-14166.

[27] (a) F. A. Scaramuzzo, E. Badetti, G. Licini, C. Zonta, Eur. J. Org. Chem 2017, 1438-1442. (b) S. Sinn, F. Biedermann, L.De Cola, Chem. Eur. J. 2017, 23, 1965-1971. (d) M. G. Costales, C. L. Haga, S. P. Velagapudi, J. L. Childs-Disney, D. G. Phinney, M. D. Disney, J. Am. Chem. Soc 2017, 139, 3446-3455. (e) A. I. Lazar, F. Biedermann, K. R. Mustafina K. I. Assaf, A. Hennig, W. M. Nau, J. Am. Chem. Soc. 2016, 138 13022-13029. (f) S. A. Kadam, K. Martin, K. Haav, L.Toom, C. Mayeux, A. Pung, P. A. Gale, J. R. Hiscock, S. J. Brooks, I. L. Kirby, N. Busschaert, I. Leito, Chem. Eur. J. 2015, 21, 5145-5160. (g) F. A. Scaramuzzo, G.Licini, C. Zonta, Chem. Eur. J. 2013, 19, 16809-16813. (h) J. P. Anzenbacher, P. Lubal, P. Bucek, M. A. Palacios, M. E. Kozelkova, Chem. Soc. Rev. 2010, 39, 3954-3979.

[28] J. Atcher, J. Solà, I. Alfonso, Org. Biomol. Chem., 2017, 15, 2013-2019. 
[29] S. P. Black, J. K. M. Sanders, A. R. Stefankiewicz, Chem. Soc. Rev. 2014, 43, 1861-1872.

[30] R. Bro, A. K. Smilde, Anal. Methods 2014, 6, 2812-2831.

[31] (a) R. Tauler, A. Izquierdo-Ridorsa, E. Casassas, Chemometr. Intell. Lab. 1993, 18, 293-300; bR. Tauler, B. Kowalski, S. Fleming, Anal. Chem. 1993, 65, 2040-2047.

[32] J. Atcher, I. Alfonso, RSC Adv. 2013, 3, 25605-25608.

[33] (a) aL. Vial, R. F. Ludlow, J. Leclaire, R. Pérez-Fernández, S. Otto, J. Am. Chem. Soc. 2006, 128, 10253-10257; (b) A. R. Stefankiewicz, M. R. Sambrook, J. K. M. Sanders, Chem. Sci. 2012, 3, 2326-2329; (c) R.
Pérez-Fernández, M. Pittelkow, A. M. Belenguer, L. A. Lane, C. V. Robinson, J. K. M. Sanders, Chem. Commun. 2009, 3708-3710.

[34] F. Savorani, G. Tomasi, S. B. Engelsen, J. Magn. Reson. 2010, 202, 190-202.

[35] J. Jaumot, A. de Juan, R. Tauler, Chemometr. Intell. Lab. 2015, 140, 112.

[36] W. H. Press, Numerical Recipes 3rd Edition: The Art of Scientific Computing, Cambridge University Press, 2007. 
Entry for the Table of Contents (Please choose one layout)

Layout 1:

\section{FULL PAPER}

A complex dynamic library of macrocycles is responsive to several chemical stimuli, such as the ionic strength, the $\mathrm{pH}$ of the medium or the presence of a biogenic polyamine as a putative substrate. The adaptive responses of the whole dynamic library were unravelled using multivariate data analysis.

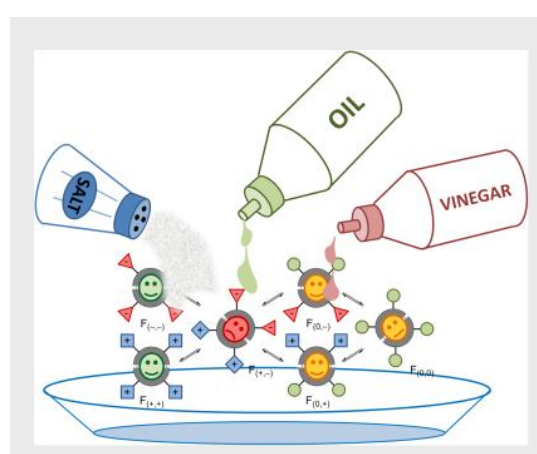

Angel M. Valdivielso, Francesc PuigCastellví, Joan Atcher, Jordi Solà, Romà Tauler and Ignacio Alfonso*

Page No. - Page No.

Unraveling the multistimuli responses of a complex dynamic system of pseudopeptidic macrocycles 